\title{
Analysis of equilibrium prices and quantities within network-structured markets applying the Lagrange function method
}

\author{
E. Aiyoshi ${ }^{\mathrm{a}}, \underline{\text { A. Maki }}{ }^{\mathrm{b}}$ and M. Nishida ${ }^{\mathrm{a}}$ \\ ${ }^{a}$ Faculty of Science and Technology, Keio University, Japan \\ ${ }^{b}$ Department of Economics, Tokyo International University, Japan \\ Email:makia@tiu.ac.jp
}

\begin{abstract}
In the modern market economic system, there are many brokers between primary producers and final producers or consumers. For example, many farmers produce vegetables and supply them to consumers. Between the farmers and consumers there are many brokers called retailers. The involved transactions are modeled by network-structured markets. Equilibrium market prices are obtained as the optimal Lagrange multipliers. The present paper analyzes changes in equilibrium prices and quantities within network-structured markets using the Lagrange function method. Then, different network structures and different specification for the sales and cost functions within the network-structured markets are analyzed.
\end{abstract}

Keywords: duality theorem for optimization, Lagrange function method, Lagrange multipliers, networkstructured market 


\section{INTRODUCTION}

In the modern market economic system, there are many brokers between primary producers and final producers or consumers. As a first example, consider the following: There are many steel makers and automobile companies using steel. Between the steel makers and automobile companies, there are many brokers that trade steel products. These brokers purchase steel from the material suppliers and each other and sell steel to other brokers and/or to automobile companies. As a second example, consider the following: There are many farmers that produce vegetables and supply them to consumers. Between the farmers and consumers there are many brokers called retailers. The involved transactions are modeled by network-structured markets.

Equilibrium market prices are obtained as the optimal Lagrange multipliers. The present paper analyzes changes in equilibrium prices and quantities within network-structured markets using the Lagrange function method (cf. Bazaraa, Sherali, Shetty, 2006). The present model includes one primary maker, two brokers, and one final producer or consumer, and it specifies sales and cost functions for the agents. Finally, simulation results using different structures of network and different specification for the sales and cost functions within the network-structured markets are analyzed.

\section{MODEL OF NEWWORK-STRUCTURED MARKET}

\subsection{Original problem}

A network system is a system of flows that are connected by arcs between nodes in the structure. For example, when we have material suppliers, brokers, and final consumers in multiple markets, the nodes are economic agents and the arcs are transactions between the agents. The arc $A(i, j)$ connecting $i$ and $j$ is taken as the market in which the transaction between buyer $i$ and seller $j$ takes place. $i$, corresponding to a node, takes values from 1 to $M$ for, namely, there are $M$ economic agents in the system. The product is transferred from $i$ to $j$. In this way the product is distributed among the markets in the network. Let us define the variables in the model:

$$
\text { (1) } \boldsymbol{x}_{i}=\left(x_{i 1}, \cdots, x_{i M}\right)^{T} \text {, }
$$

where $x_{i j}$ is a transaction volume for sales from $i$ to $j$ and $\boldsymbol{x}_{i}$ is the total sales volume for agent $i$.

(2) $\boldsymbol{y}_{i}=\left(y_{1 i}, \cdots, y_{M i}\right)^{T}$,

where $y_{j i}$ is the transaction volume for purchases by $i$ from $j$.

(3) $f_{i}\left(\boldsymbol{x}_{i}, \boldsymbol{y}_{i}\right):$ profit function for agent $i$

(4) $u:$ the amount of production

(5) $v$ : the amount of consumption $(u=v)$

The objective function of the model is to maximize the sum of the profits over all nodes of the network structure:

$$
\begin{gathered}
\max _{\left\{\boldsymbol{x}_{i}\right\},\left\{\boldsymbol{y}_{i}\right\}} \sum_{i=1}^{M} f_{i}\left(\boldsymbol{x}_{i}, \boldsymbol{y}_{i}\right) \\
\text { subject to } \sum_{(i, j) \in A} x_{i j}-\sum_{(k, i) \in A} y_{k i}=0, \quad i=1, \cdots, M \\
x_{i j}=y_{i j},(i, j) \in A \\
x_{i j}, y_{i j} \geq 0,(i, j) \in A,
\end{gathered}
$$

where $(2.1 \mathrm{~b})$ is the equality constraint regarding the sum of inflow and outflow at each node, $(2.1 \mathrm{c})$ is the equilibrium condition between the amount of demand and supply in each market, and (2.1d) is the nonnegativity constraint for the flow amounts. The objective function seeks the optimal value of $\left\{x_{i j} \mid(i, j) \in A\right\}$ to maximize the sum of the profit within the network structure under constraints from (2.1b) to $(2.1 \mathrm{~d})$. 
Aiyoshi et al., Analysis of equilibrium prices and quantities within network-structured markets applying the Lagrange function method

Figure 1 shows an example network structure.

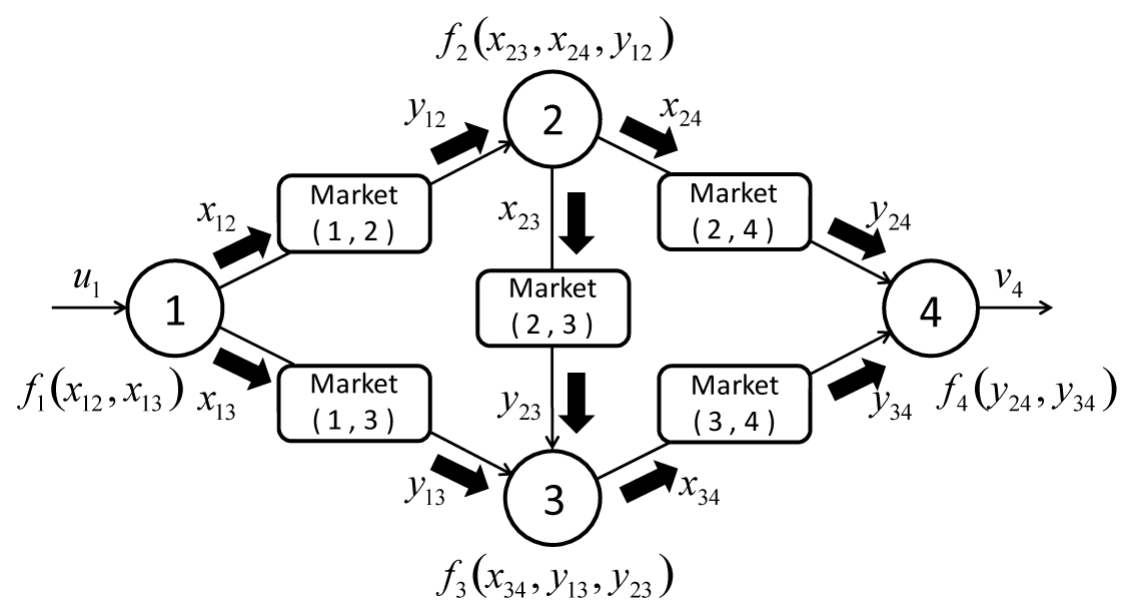

\subsection{Dual problem}

Based on equation (2.1) as the original problem, a network model is introduced in this section by use of the Lagrange function method and dual theory (in Appendix). The Lagrange function corresponding to (2.1a) is

$$
L\left(\left\{\boldsymbol{x}_{i}\right\},\left\{\boldsymbol{y}_{i}\right\} ;\left\{\varphi_{i}\right\}\right)=\sum_{j=1}^{M} f_{i}\left(\boldsymbol{x}_{i}, \boldsymbol{y}_{i}\right)+\sum_{(i, j) \in A} \varphi_{i j}\left(x_{i j}-y_{i j}\right)
$$

where the Lagrange multiplier corresponding to node $(i, j)$ is $\varphi_{i j}$ and $\varphi_{i}=\left(\varphi_{i 1}, \cdots, \varphi_{i M}\right)^{T}$. The Lagrange problem corresponding to the original problem of (2.1) is

$$
\begin{aligned}
& \max _{\left\{\boldsymbol{x}_{i}\right\},\left\{\boldsymbol{y}_{i}\right\}} L\left(\left\{\boldsymbol{x}_{i}\right\},\left\{\boldsymbol{y}_{i}\right\},\left\{\varphi_{i}\right\}\right) \\
& \text { subject to } \\
& \sum_{(i, j) \in A} x_{i j}-\sum_{(k, i) \in A} y_{k i}=0, \quad i=1, \cdots, M \\
& x_{i j}, y_{i j} \geq 0,(i, j) \in A
\end{aligned}
$$

Here, the Lagrange function (2.2) is

$$
\begin{aligned}
& L\left(\left\{\boldsymbol{x}_{i}\right\},\left\{\boldsymbol{y}_{i}\right\},\left\{\varphi_{i}\right\}\right) \\
& =\sum_{i=1}^{M} f_{i}\left(\boldsymbol{x}_{i}, \boldsymbol{y}_{i}\right)+\sum_{(i, j) \in A} \varphi_{i j} x_{i j}-\sum_{(i, j) \in A} \varphi_{i j} y_{i j} \\
& =\sum_{i=1}^{M} f_{i}\left(\boldsymbol{x}_{i}, \boldsymbol{y}_{i}\right)+\sum_{i=1}^{M} \sum_{(i, j) \in A} \varphi_{i j} x_{i j}-\sum_{i=1}^{M} \sum_{(k, j) \in A} \varphi_{k j} y_{k j} \\
& =\sum_{i=1}^{M}\left\{f_{i}\left(\boldsymbol{x}_{i}, \boldsymbol{y}_{i}\right)+\sum_{(i, j) \in A} \varphi_{i j} x_{i j}-\sum_{(k, j) \in A} \varphi_{k j} y_{k j}\right\}
\end{aligned}
$$

Therefore, the Lagrange problem (2.3) becomes the maximization problem for each node:

$$
\begin{array}{cl}
\max _{\left(\boldsymbol{x}_{i}, \boldsymbol{y}_{i}\right)}\left\{f_{i}\left(\boldsymbol{x}_{i}, \boldsymbol{y}_{i}\right)+\sum_{(i, j) \in A} \varphi_{i j} x_{i j}-\sum_{(k, j) \in A} \varphi_{k j} y_{k j}\right\} \\
\text { subject to } & \sum_{(i, j) \in A} x_{i j}-\sum_{(k, i) \in A} y_{k i}=0 \\
& x_{i j} \geq 0,(i, j) \in A \\
& y_{i j} \geq 0,(k, i) \in A
\end{array}
$$


Aiyoshi et al., Analysis of equilibrium prices and quantities within network-structured markets applying the Lagrange function method

As the Lagrange multiplier $\varphi_{i j}$ is the market transaction price at the node $(i, j)$, the evaluation function in (2.5a) includes not only the profit but also the difference of the sales and the cost. This is because under the condition of the Lagrange multiplier $\varphi_{i}=\left(\varphi_{i 1}, \cdots, \varphi_{i M}\right)^{T}$ as the fixed market prices, the agents decide the selling and buying transactions at each node.

On the maximization of Lagrange problem (2.5) for each $i$, the dual function, $v\left(\left\{\varphi_{i}\right\}\right)$, is defined as

$$
v\left(\left\{\varphi_{i}\right\}\right)=\sum_{m=1}^{M} v_{i}\left(\varphi_{i}\right)
$$

and the dual of the original problem becomes

$$
\min _{\left\{\varphi_{i}\right\}} v\left(\left\{\varphi_{i}\right\}\right)
$$

When the solution to (2.5) is unique, then the solution can be obtained by solving problem (2.7) by the steepest descent method. The process to obtain the solution updates using

$$
\varphi_{i j}(k+1)=\varphi_{i j}(k)-\varepsilon\left(x_{i j}^{*}\left(\varphi_{i}(k)\right)-y_{i j}^{*}\left(\varphi_{i}(k)\right)\right),(i, j) \in A,
$$

where $\left(x_{i j}^{*}\left(\varphi_{i}(k)\right), y_{i j}^{*}\left(\varphi_{i}(k)\right)\right)$ is the solution of (2.5) given the Lagrange multiplier $\varphi_{i}(k)$.

When the second term of the right-hand side of (2.8) becomes zero, the optimal market prices have been determined. The solution set of $\left(\boldsymbol{x}_{i}^{*}\left(\varphi_{i}^{*}\right), \boldsymbol{y}_{i}^{*}\left(\varphi_{i}^{*}\right)\right)$ obtained for (2.5) becomes the optimal solution for the original problem of (2.1), where the solution set gives the volume of transactions in the network.

The calculation procedure is

Step 1: The initial values of $\varphi_{i j}(0)$ is given corresponding to the each node pair $(i, j)$, and $k$ is set as 0 .

Step 2: Taking given $\varphi_{i}(k)$, solving the $M$-Lagrange problems of (2.5) to get a set of $\left(\boldsymbol{x}_{i}(k), \boldsymbol{y}_{i}(k)\right)$.

Step 3: Updating the prices using equation (2.8) and the set of $\left(\boldsymbol{x}_{i}(k), \boldsymbol{y}_{i}(k)\right)$ until the condition of $\boldsymbol{x}_{i}(k)=\boldsymbol{y}_{i}(k)$ are satisfied. If the constraints cannot be satisfied, return to step 2 .

\subsection{Simulation}

The profit function is specified as a quadratic form as

$$
f_{i}\left(\boldsymbol{x}_{i}, \boldsymbol{y}_{i}\right)=\sum_{j}-c_{i j}\left(x_{i j}-a_{i j}\right)^{2}+\sum_{k}-d_{k i}\left(y_{k i}-b_{k i}\right)^{2},
$$

where $a_{i j}, b_{i j}, c_{i j}$ and $d_{i j}$ are parameters.

Using this equation, a solution of the simulation model will be obtained. First we will denote the initial conditions. The common conditions for the simulation are as follows: The solution method is the steepest descent method with penalty terms: Euler difference in the steepest descent method has a step size of 1.00 $\times 10^{-4}$; the maximum number of iteration is 50,000 ; the step size for $(2.8)$ is $\mathcal{E}=0.50$; and the initial Lagrange multiplier for each node is 0.00 . The parameters $a_{i j}, b_{i j}, c_{i j}$ and $d_{i j}$ are different for each simulation.

Tables 2.1 to 2.5 show the parameter values for $a_{i j}, b_{i j}, c_{i j}$ and $d_{i j}$ for each node pair along with the simulation results for the sales and costs of agents. Table 2.1 is the benchmark case, in which all the parameter values for $a_{i j}, b_{i j}, c_{i j}$ and $d_{i j}$ are the same for all agent pairs. This means sales and const functions are the same for all agents. 
Aiyoshi et al., Analysis of equilibrium prices and quantities within network-structured markets applying the Lagrange function method

Table 2.1 Benchmark Case

Panel (a): Parameters

\begin{tabular}{|c|c|c|c|c|c|c|}
\hline arc & $a_{i j}$ & $b_{i j}$ & $c_{i j}$ & $d_{i j}$ & $\varphi_{i}$ & $x_{i j}=y_{i j}$ \\
\hline$(1,2)$ & 3.0 & 6.0 & 1.0 & 1.0 & 1.95 & 5.0 \\
\hline$(1,3)$ & 3.0 & 6.0 & 1.0 & 1.0 & 1.95 & 5.0 \\
\hline$(2,4)$ & 3.0 & 6.0 & 1.0 & 1.0 & 3.95 & 5.0 \\
\hline$(3,4)$ & 3.0 & 6.0 & 1.0 & 1.0 & 3.95 & 5.0 \\
\hline
\end{tabular}

Panel (b): Sales, cost and profit

\begin{tabular}{|c|c|c|c|c|}
\hline node (agent) & 1 & 2 & 3 & 4 \\
\hline sales & 19.5 & 19.75 & 19.75 & \\
\hline cost & & 9.75 & 9.75 & 39.5 \\
\hline profit & & 10.0 & 10.0 & \\
\hline
\end{tabular}

In the benchmark case shown in Table 2.1, the market price for arcs $(1,2)$ and $(1,3)$ are both 1.95 and those for $(2,4)$ and $(3,4)$ are 3.95 . The sale of the producer (agent 1$)$ to the agents 2 and 3 is 19.5 , and the cost of the final retailer (agent 4 ) is 39.5 for the same volume of 10 units. The total profit of the structure is 20.0 which is divided equally between agents 2 and 3 .

The next simulation considers when an arc between agents 2 and 3 exists. The brokers buy and sell not only for agent 4 but also buy and sell between agents 2 and 3 each other. In this case, the parameters of sales and cost functions are identical but the market prices for arcs $(1,2)$ and $(1,3)$ are different. The parameter values and the simulation results are shown in Table 2.2.

Table 2.2 Existence of the arc between 2 and 3 Panel (a): Parameters

\begin{tabular}{lcccccc} 
arc & $a_{i j}$ & $b_{i j}$ & $c_{i j}$ & $d_{i j}$ & $\varphi_{i}$ & $x_{i j}=y_{i j}$ \\
\hline$(1,2)$ & 3.0 & 5.0 & 1.0 & 1.0 & 1.95 & 6.0 \\
$(1,3)$ & 3.0 & 5.0 & 1.0 & 1.0 & -2.05 & 4.0 \\
$(2,3)$ & 3.0 & 5.0 & 1.0 & 1.0 & 1.95 & 2.0 \\
$(2,4)$ & 3.0 & 5.0 & 1.0 & 1.0 & 5.95 & 4.0 \\
$(3.4)$ & 3.0 & 5.0 & 1.0 & 1.0 & 1.95 & 6.0
\end{tabular}

Panel (b): Sales, cost and profit

\begin{tabular}{|c|c|c|c|c|}
\hline node (agent) & 1 & 2 & 3 & 4 \\
\hline sales & 3.5 & 27.7 & 11.7 & \\
\hline $\operatorname{cost}$ & & 11.7 & -4.3 & 35.5 \\
\hline profit & & 16.0 & 16.0 & \\
\hline
\end{tabular}


Aiyoshi et al., Analysis of equilibrium prices and quantities within network-structured markets applying the Lagrange function method

In the simulation, the transaction price between agents 1 and 3 is -2.05 indicating agent 1 sells 4 units of the commodity to the agent 3 at this price in order to maximize the profit in the network structure. In the simulation the total sales for agent 1 is 3.5 , and the total cost for agent 4 is 35.5 . Total profit is again divided equally between agents 2 and 3 .

When the parameter $b_{i j}$ for the cost functions varies among the agents, the total profit is not divided equally between agents 2 and 3 . Table 2.3 shows the parameter values and the resulting sales, costs and profits for the four agents.

Table 2.3 The cost functions varies among the agents (parameter $b_{i j}$ )

Panel (a): Parameters

\begin{tabular}{|c|c|c|c|c|c|c|}
\hline $\operatorname{arc}$ & $a_{i j}$ & $b_{i j}$ & $c_{i j}$ & $d_{i j}$ & $\varphi_{i}$ & $x_{i j}=y_{i j}$ \\
\hline$(1,2)$ & 3.0 & 8.0 & 1.0 & 1.0 & 4.45 & 5.75 \\
\hline$(1,3)$ & 3.0 & 5.0 & 1.0 & 1.0 & 1.45 & 4.25 \\
\hline$(2,4)$ & 3.0 & 8.0 & 1.0 & 1.0 & 5.45 & 5.75 \\
\hline$(3,4)$ & 3.0 & 5.0 & 1.0 & 1.0 & 2.45 & 4.25 \\
\hline
\end{tabular}

Panel (b): Sales, cost and profit

\begin{tabular}{|c|c|c|c|c|}
\hline node (agent) & 1 & 2 & 3 & 4 \\
\hline sales & 31.75 & 10.4125 & 31.3375 & \\
\hline cost & & 6.1625 & 25.5875 & 41.75 \\
\hline profit & & 4.25 & 5.75 & \\
\hline
\end{tabular}

As shown, the profits for agents 2 and 3 differ because the cost functions differ.

The next case simulates differences in sales functions among agents. This is explicitly specified by the parameter $c_{i j}$ as shown in Table 2.4 .

Table 2.4 The sales functions varies among the agents (parameter $c_{i j}$ )

Panel (a): Parameters

\begin{tabular}{|c|c|c|c|c|c|c|}
\hline $\operatorname{arc}$ & $a_{i j}$ & $b_{i j}$ & $c_{i j}$ & $d_{i j}$ & $\varphi_{i}$ & $x_{i j}=y_{i j}$ \\
\hline$(1,2)$ & 3.0 & 6.0 & 2.0 & 1.0 & 2.75 & 4.60 \\
\hline$(1,3)$ & 3.0 & 6.0 & 1.0 & 1.0 & 1.15 & 5.40 \\
\hline$(2,4)$ & 3.0 & 6.0 & 2.0 & 1.0 & 6.35 & 4.60 \\
\hline$(3,4)$ & 3.0 & 6.0 & 1.0 & 1.0 & 4.75 & 5.40 \\
\hline
\end{tabular}


Aiyoshi et al., Analysis of equilibrium prices and quantities within network-structured markets applying the Lagrange function method

Panel (b): Sales, cost and profit

\begin{tabular}{lllll} 
& & & \\
node $($ agent & 1 & 2 & 3 & 4 \\
\hline sales & 20.48 & 29.21 & 25.65 & \\
cost & & 12.65 & 7.83 & 54.86 \\
profit & & 16.56 & 17.82 &
\end{tabular}

When sales functions are not symmetric, profit is not distributed equally between agents 2 and 3 . The final simulation uses differing cost functions among the agents, which is explicitly specified in parameter $d_{i j}$ as shown in Table 2.5.

Table 2.5 The cost functions varies among the agents (parameter $d_{i j}$ )

Panel (a): Parameters

\begin{tabular}{lllllll} 
& & & & & \\
- & $a_{i j}$ & $b_{i j}$ & $c_{i j}$ & $d_{i j}$ & $\varphi_{i}$ & $x_{i j}=y_{i j}$ \\
- \hdashline$(1,2)$ & 3.0 & 6.0 & 1.0 & 2.0 & 3.15 & 5.20 \\
$(1,3)$ & 3.0 & 6.0 & 1.0 & 1.0 & 2.35 & 4.80 \\
$(2,4)$ & 3.0 & 6.0 & 1.0 & 2.0 & 4.35 & 5.20 \\
$(3,4)$ & 3.0 & 6.0 & 1.0 & 1.0 & 3.55 & 4.80
\end{tabular}

Panel (b): Sales, cost and profit

\begin{tabular}{|c|c|c|c|c|}
\hline node (agent) & 1 & 2 & 3 & 4 \\
\hline sales & 23.53 & 22.62 & 17.04 & \\
\hline cost & & 16.38 & 11.28 & 39.66 \\
\hline profit & & 6.24 & 5.76 & \\
\hline
\end{tabular}

\section{CONCLUSION}

The present paper analyzes changes in optimal market price in a network structure. Using the Lagrange function method, market prices distributed on network- structured markets are derived easily as the solutions to the dual problem of the original profit maximization with respect to transaction quantities.

\section{ACKNOWLEDGMENT}

The authors are grateful to Michael McAleer and Les Oxley. This research is supported by the JSPS research grant-in-aid.

\section{REFERENCES}

Bazarra, M.S., H.D. Sherali and C.M. Shetty (2006), Nonlinear Progamming: Theory and Algorithms, Thrid Edition, John Wiley: Hoboken, New Jersey.

Dixit, A.K. and V. Norman (1980), Theory of International Trade: A Dual, General Equilibrium Approach, Cambridge University Press: Beccles and London.

Dixit, A.K. (1990), Optimization in Economic Theory, Second Edition, Oxford University Press: Oxford. Iri, M. (1969), Network Flows, Transportation and Scheduling - Theory and Algorithm, Academic Press. Jamshidi, M. (1983), Large-Scale Systems: Modeling and Control, North-Holland: New York. 